\title{
A Catalog of Speculative Playful Urban Technology Ideas
}

\author{
Exploring the Playful Potential of Smart Cities
}

\author{
Ferran Altarriba Bertran \\ Social Emotional Technology Lab, \\ University of California Santa Cruz \\ ferranaltarriba@gmail.com \\ Ivy Chen \\ Social Emotional Technology Lab, \\ University of California Santa Cruz \\ ichen9@ucsc.edu
}

\author{
Jared Duval \\ Social Emotional Technology Lab, \\ University of California Santa Cruz \\ jduval@ucsc.edu \\ Victor Dong \\ Social Emotional Technology Lab, \\ University of California Santa Cruz \\ vldong@ucsc.edu
}

\author{
Laura Bisbe Armengol \\ Escola de Realització Audiovisual i \\ Multimèdia, Universitat de Girona \\ laura.bisbe@gmail.com \\ Binaisha Dastoor \\ Social Emotional Technology Lab, \\ University of California Santa Cruz \\ bdastoor@ucsc.edu
}

\author{
Adrià Altarriba Bertran \\ Independent designer \\ aaltarribab@gmail.com
}

\author{
Katherine Isbister \\ Social Emotional Technology Lab, \\ University of California Santa Cruz \\ kisbiste@ucsc.edu
}

\begin{abstract}
In this paper, we present an annotated portfolio of speculative playful urban technology concepts. Our portfolio builds on and illustrates in designerly ways the findings from our research into the playful potential of urban spaces. We begin by describing two Situated Play Design [1] interventions where we identified play potentials of urban spaces, i.e., playful things that people already do in public spaces and might have socio-emotional value. We suggest that these play potentials could inspire the design of future smart city technology that pays more attention to the social, emotional, and cultural role of urban spaces in human life. To show that potential and foreground the inspirational value of our findings, we present a catalog of speculative design ideas that embody the design directions and play potentials we identified in our research. We hope to contribute to an existing and exciting body of work aimed at inspiring the design of increasingly playful and socio-emotionally sustainable urban technology.
\end{abstract}

\section{CCS CONCEPTS}

- Human-centered computing $\rightarrow$ Interaction design; Interaction design process and methods; Contextual design.

\section{KEYWORDS}

Play, Smart cities, Urban technology, Situated Play Design, Fun, Speculative design

Permission to make digital or hard copies of all or part of this work for personal or classroom use is granted without fee provided that copies are not made or distributed for profit or commercial advantage and that copies bear this notice and the full citation on the first page. Copyrights for components of this work owned by others than the author(s) must be honored. Abstracting with credit is permitted. To copy otherwise, or republish, to post on servers or to redistribute to lists, requires prior specific permission and/or a fee. Request permissions from permissions@acm.org.

Mindtrek '21, June 01-03, 2021, Tampere/Virtual, Finland

(c) 2021 Copyright held by the owner/author(s). Publication rights licensed to ACM. ACM ISBN 978-1-4503-8514-5/21/06 . \$ \$15.00

https://doi.org/10.1145/3464327.3464374
ACM Reference Format:

Ferran Altarriba Bertran, Jared Duval, Laura Bisbe Armengol, Ivy Chen, Victor Dong, Binaisha Dastoor, Adrià Altarriba Bertran, and Katherine Isbister. 2021. A Catalog of Speculative Playful Urban Technology Ideas: Exploring the Playful Potential of Smart Cities. In Academic Mindtrek 2021 (Mindtrek '21), fune 01-03, 2021, Tampere/Virtual, Finland. ACM, New York, NY, USA, 12 pages. https://doi.org/10.1145/3464327.3464374

\section{INTRODUCTION}

Smart cities are often presented through the lens of increasing urban efficiency [6], optimizing infrastructure [42], and spurring the economy [19]. Less attention is paid to our cities' socio-cultural fabric [14][43], especially in commercial implementations [31]. We see that as a missed opportunity when it comes to cultivating stimulating urban spaces where humans can flourish. Inspired by existing work exploring less techno-centric (e.g., [5][45][51][56]) and increasingly playful (e.g., [30][47][53][61][64]) urban futures, here we investigate the potential of play to contribute to the socio-cultural sustainability of urban spaces.

Research suggests that cities should be far more than productive [47][61]: while there is value in designing urban tech that makes our cities more efficient, designers should also pay attention to the social, emotional, and cultural qualities of the urban space. Here we contribute to an emerging body of work that explores how to do that by adding an element of play to our streets (e.g., the designs presented in [30] and [47], or those described in [64]). We explore the inherent playful potential of urban spaces and speculate upon how to design interactive technology that helps us to realize it.

In this paper, we present the interim outcomes of a design-led exploration investigating forms of play that might be desirable in urban spaces. Our contribution is two-fold: First, a list of play potentials [1] of urban spaces (i.e., forms of urban play that are grounded in people's existing urban practices and that might have socio-emotional value). Second, a collection of speculative playful urban technology ideas that build on and embody those play potentials, illustrating how they might inspire design. Overall, we aim to empower designers to embrace increasingly playful and 
socio-emotional approaches to urban innovation. Here we provide inspirational provocations that can facilitate that move

\section{BACKGROUND}

\subsection{Smart City Innovation: Beyond Productivity}

With every generation, technology weaves its way more deeply into our lives, and each time we must remake the choice to care about lived experience. Smart homes represented one such inflection point a couple of decades ago: in response to the increasing presence of digital technology in our lives, the Equator Project [24] explored how not to lose sight of lived experience in the face of productivityfocused technology trends. Today, chiefly in the industry sector [31], smart cities present similar challenges: while promising increased competitiveness and quality of life [7], innovation in this space often favors techno-centric rhetoric that privileges agendas such as increasing urban efficiency [6], optimizing infrastructure [42], or spurring economic activity [19]. Less attention is put to social intelligence, cultural artifacts, or environmental attributes [43]-all key components of socio-emotionally sustainable urban entanglements.

Alternative approaches to urban innovation exist, e.g.: humancentered smart city design [5], democratic [45] and inclusive [51] urban planning, bottom-up urban innovation [14], or sustainable smart city initiatives [56], among others. Here we contribute to a particular subset of this research space that approaches urban innovation through the lens of playfulness: the so-called playable city [47]. Building on, and inspired by recent research in this space (e.g., [30][53][61][64]), we argue that cities should be far more than efficient; they should also be socially rewarding, culturally stimulating, and emotionally rich. We highlight the importance of embracing those values if we want to design technology-mediated urban spaces that are socio-emotionally sustainable. Play can be a way of facilitating that move-a lens through which we can reclaim the playful, socio-cultural function of our cities and resemantize [64] them so they respond to our need for everyday play.

\subsection{The Socio-Emotional Relevance of Play}

Humans are not productivity machines-we are motivated by pleasure, by social and emotional connection, by agency, and by positive feelings [12]. We argue that if cities fail to afford these experiences, they can become unwelcoming. Fun and joy are not optional qualities when it comes to urban spaces-they are deeply necessary, as we know from Jane Jacobs' work [15] on what makes cities places to thrive. Cities are made up of moment-to-moment passing interactions between humans, and as such, they should cater to our social, emotional, and cultural needs.

Play can be a way to bring these properties into the smart city systems we build: it is a fundamental human need [10][12][13][34] [54] that enriches our experience of the world we live in. Sharp and Thomas call this the eudaimonic function of play: even if it does not yield materially productive outcomes, it can be considered socio-emotionally productive [54]. Here we argue for the relevance of play in the context of urban innovation, looking at research from play studies, play design, and playable cities to highlight three highly relevant socio-emotionally desirable qualities of play:
Play brings joy to situations that might otherwise be unstimulating: it generates positive emotions because it speaks to our inner desire for joy and stimulation [10]. It is commonly said that, when playing, "time flies": play puts us in a state of flow where we are deeply immersed in, and profoundly enjoy, whatever we are doing [18]. Though occasional boredom can contribute to our well-being [65], play can help us to reframe situations that are annoying or tedious [10]: it brings about fun and laughter and allows us to thrive. As [30][47][61] show, playful technology can help us to season countless playless situations we experience on a daily basis by turning urban spaces into ephemeral playgrounds. It can create porous circles of play [59] that, while not removing us from the situation, afford "entertaining and healthy experiences that improve [our] quality of life" [47] and give us chances to let go of unnecessary feelings of boredom and frustration-feelings that are unfortunately not uncommon in our ordinary urban engagements.

Play can help us to experience a feeling of agency: it allows us to choose, act, and express ourselves in ways that feel meaningful [54][55]. That can be very valuable in a world where we are increasingly busy and reliant on larger structures, to the extent that we feel a lack of control of our life [58][62]. Play empowers us to act upon the world surrounding us [55] and cultivates creative ideation [66]. That is, in part, because play is both appropriative and disruptive: it takes over the context where it happens and challenges the state of affairs [55]. Being playful allows us to bring freedom to a context without disrupting it completely [55], setting the right conditions to create [57]. Recent research has shown that those are desirable qualities in the context of urban spaces and that playful urban tech can entice people to play an active part in the ongoing development of their city [21].

Play supports social affordances [36], which is highly relevant given that social interaction is key to human flourishing [35]-even more so in a contemporary society that suffers from an increasing lack of meaningful social connection [37]. Some argue that technology use might contribute to that issue (e.g., through screen-based interactions that distract people from experiencing co-located social interaction [33]). But technology does not necessarily need to isolate us-it has the capacity (and, we argue, the responsibility) of enriching our social lives. Play can support that, as it is known to have "a positive impact on the wellbeing of both individuals and groups" [34]. Playful technology can help us to bring people together [35], physically or virtually, a quality that is desirable in the urban space as it can help to enrich people's urban experiences and support collective bottom-up city-making [40][53].

These experiential qualities (joy, agency, and social connection) respond to important societal values. We argue they should be carefully considered when designing (for) the urban space-they are desirable social goods that can positively affect the well-being of both individuals and groups. While these qualities are not exclusively present in playful experiences, play can help us access them-in and beyond the urban space. In a world where technology is increasingly present, designers should cultivate those kinds of experiences. Technology can undoubtedly help us be more productive, but it should also augment the experiential texture of our lives. With the work presented in this paper, we hope to contribute 
to an ongoing move towards more playful and socio-emotionally sensitive urban innovation.

\subsection{Urban Technology, Play, and HCI}

In HCI, a plethora of design and research work has explored the intersection of play and smart cities-a space that is often referred to as the playable city [47]. Within this space, different approaches exist. For example, location-based games use urban spaces as physical landscapes within which virtual game worlds emerge. Whether in the form of research designs (e.g., [29]) or commercial products (e.g., Pokémon Go [46]), these games typically present alternate realities that take place outside of the player's ordinary routine-gameful, entertainment-focused virtual worlds that hardly interface with the player's ordinary urban practices.

Another set of playable city interventions are those that gamefully augment urban activity to support productive outcomes what [64] calls urban gamification. For example, a serious game to reduce energy consumption in smart buildings [27], or a gamified app to optimize urban transportation [22]. In some cases, those interventions are playful rather than gameful [41], e.g., a set of piano stairs to promote healthier habits in a public space [50]. Unlike location-based games, these designs strongly adapt the gameplay experience to people's ordinary, non-play activity. Further, they are characterized by their aim to support productive, measurable goals rather than to simply enrich people's experience of their city-an agenda that is central to our work.

Another type of playful urban tech are civic games that promote playful citizenship [30]-or, as [21] puts it, games that "shift the attention from smart cities to smart citizens" by playfully empowering the latter to actively engage in city-making. Unlike location-based games, civic games relate strongly to people's ordinary practices; unlike gamified apps or serious games, they are meant to enhance a city's socio-cultural dimension rather than its productive capacity. For example, Commons is a game about filing claims about community needs by gathering evidence on city streets [38]. We are inspired by how those games support rich socio-cultural engagements between citizens and their urban space.

Finally, we see playful urban designs that are not games per se but technology-mediated invitations to engage in free-form urban play. [3] characterizes them as Technology for Situated \& Emergent Play, i.e., "technology design that supports playful engagement that emerges interwoven with our everyday activities outside leisure, and that enriches these activities with socio-emotional value." For example, SelfieCafe fosters social interaction between community members by allowing them to take a selfie and share it on a large display [52]; spread.gun allows people to sabotage advertisement screens and "reclaim" them with custom messages [25]; or Urbanimals [39] and Hello Lamp Post [49] allow people to interact with urban infrastructure playfully, let it be physically or verbally, in ways that do not necessarily bring about any productive gain. These works enable a resemantization of the urban space, allowing play to "infiltrate several contexts and spaces, and to propose new meanings, new constraints, new strategies, and new motivations" [64]. We are inspired by how they invite people to spontaneously reframe ordinary spaces into arenas for exploration, creativity, communitybuilding, and shared ephemeral joy.
Despite their differences, those four types of playful and gameful urban technology share a focus on infusing aspects of people's urban endeavors with an element of playfulness-or, as [64] puts it, a "desire to rewrite the city, to reshape it, to engrave oneself in it, to renew it by resorting to the energy and the ability to motivate people that emanates from play." Insofar as they propose experiences that (in different ways and to different extents) intertwine with people's ordinary urban activity, the playfulness they afford must be contextually meaningful. This paper argues for playable cities where play is smoothly and carefully integrated into people's daily lives. We hope that our work inspires designers interested in strengthening the palette of design exemplars bridging play, technology, and urban design.

\section{CHASING PLAY POTENTIALS OF URBAN SPACES}

Designing technology that enables playfulness to permeate our daily lives-in and beyond the urban space-poses methodological challenges. One of them is embracing the idiosyncrasies of users and their contexts to ensure that the experience afforded by the technology intertwines well with the non-play situation at hand [8]. Recent works in HCI (e.g., [60]) have called for new methods that support designers to deal with the contextual and emergent nature of playful engagement in non-play scenarios. In response to those calls, [1] proposed Situated Play Design (SPD) as a design approach that focuses on "chasing play potentials" and using them to inspire the core components of a contextually meaningful play experience. Play potentials are spontaneous manifestations of playful engagement that emerge in the wild-playful things people already do in their day-to-day, and that as such carry valuable knowledge about the kinds of playful experiences they enjoy [1].

Here we position SPD as a useful approach to designing playful tech that enriches people's interactions with(in) their city in ways that enable [64]'s aforementioned resemantization of the urban space. SPD focuses designers' attention on playful experiences that people already enjoy in a particular context-ones that are likely meaningful to them and that, as such, may have inspirational potential. By chasing play potentials in the urban space, designers can uncover play forms that people already find meaningful and enjoyable. Those play potentials can then be used as starting points to inspire urban technology design, leading to ideas that align well with playful practices citizens already feel excited about. Such an approach can help designers to focus their ideation on types of playful experiences and encounters that resonate with a city's socio-cultural fabric and thereby contribute to realize (rather than disrupt) the city's inherent playful potential. Below, we describe how we used two SPD interventions to explore the playful potential of urban spaces and present the play potentials we identified.

\subsection{Intervention \# 1: Scraping Social Media}

In our first intervention, inspired by [4], we created a collection of social media posts (made by other users) and examined it to identify recurrent forms of playful engagement within the public space. Our aim was to address the first stage of SPD: chasing play potentials we could then use as inspirational starting points for design. For 5 weeks, 6 researchers collected 383 posts, focusing on Instagram and 


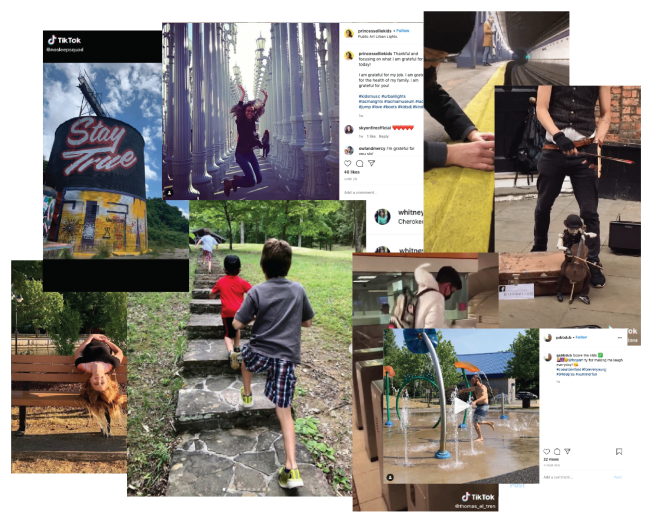

Figure 1: Examples of posts in our collection displaying forms of playful engagement with(in) the public space.

TikTok due to their leisurely and visual nature. We did not focus on a specific location or a specific idea of what play might mean. If we deemed a post featured playful urban behavior, we included it; where there was a doubt, the post was discussed among the team. Posts made by professional social media influencers were excluded from the onset to avoid unspontaneous content. Posts were stored in a spreadsheet, including a link to the post, a short description, the publication date, and (if needed) a note indicating what was inspirational about them. We then used inductive thematic analysis [32] to explore our collection and identify play potentials. After two rounds of refining our emergent themes (i.e., the forms of playful behavior we kept seeing come up in the posts, such as "leaving messages for other people to find"), we settled on a final set of play potentials. We then analyzed the entire dataset, assigning one or more play potentials to each post. The result was a list of 18 urban play potentials ${ }^{1}$ (i.e., ways in which people engage playfully within the public space) each instantiated by several examples of social media posts.

\subsection{Intervention \#2: Play \& Culture Workshop}

In our second intervention, we turned to culture and traditions to seek forms of emergent play embedded in rituals and games practiced in the public space-again, to identify play potentials we could use to nurture our design process. To focus our exploration, we focused on traditions from a specific cultural background: Catalonia, the region around the city of Barcelona. Inspired by recent works exploring culture as a source of play potentials [1][16], we invited 13 participants to a 3-hour online workshop where we explored urban traditions from their culture to uncover forms of urban play that were meaningful to them. Participants' professional backgrounds were relevant to play and/or urban innovation: we invited two gamification consultants, three experts on folk games, a videogame developer, an interaction designer, a communication designer, a philosopher, an architect, and a journalist specialized in emergent technology.

Before the workshop, we carefully crafted a Miro collaboration environment $^{2}$ (Figure 2) to facilitate the conversations. We populated the board with a set of playful urban traditions from our

\footnotetext{
${ }^{1}$ Our collection of social media posts \& play potentials: http://bit.ly/postspotentials

${ }^{2}$ The workshop Miro board: http://bit.ly/miroboardwkshp
}

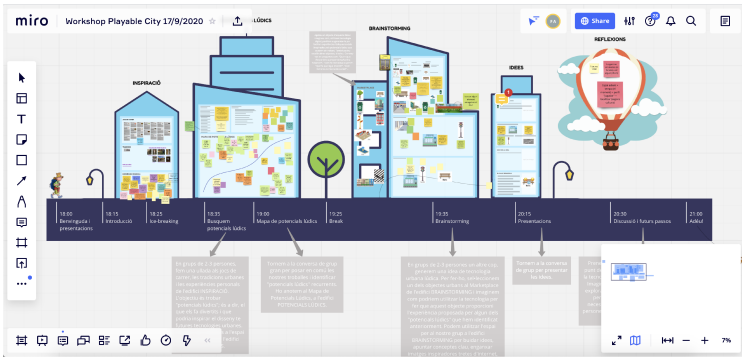

Figure 2: Screenshot of the Miro board, taken after the workshop. It features all the contributions made by participants, as well as the base structure we set up in preparation for the workshop.

previous research and shared it with participants a week in advance so they could familiarize themselves with the traditions. We also invited participants to add new traditions to the collection if they wanted to. During the workshop, we used the Miro board to present, discuss, and visualize our ideas. First, we examined the collection of urban traditions to identify interesting forms of playful engagement. Then, we brought our findings into a map of play potentials of urban spaces. Next, we brainstormed how urban infrastructure (e.g., a bench) might afford technology-mediated experiences inspired by one or more of the play potentials. To close, we built on those speculative ideas to discuss the relevance of the play potentials found throughout the workshop and, more broadly, of our playful approach to smart city innovation. After the workshop, two researchers analyzed both the annotations on the Miro board and the workshop recordings to synthesize participants' main contributions. The result was a list of 9 urban play potentials inspired by urban traditions, each instantiated by at least one urban game, ritual, or personal experience.

\subsection{Results: Play Potentials of Urban Spaces}

Once we completed the two SPD interventions, a researcher did a second round of analysis to combine the two resulting sets of play potentials into a unified list. The aim was to: (1) synthesize our findings into a unique list of play potentials that was manageable and actionable-one that was not too long, complex, or overwhelming to be used as starting point for ideation; and (2) focus on and surface those aspects of our findings that were most inspirational, to orient designers' attention towards how those forms of playful urban engagement could give rise to new kinds of urban technology.

To combine the play potentials from both interventions, we clustered them by affinity, looking at the similarities between the play forms they highlighted and thinking about how they might inspire designers to imagine increasingly playful smart city futures. To ensure that the combined list accurately represented the results from our two interventions, we shared the second-wave analysis with researchers involved in both interventions and gave them a chance to dispute it.

Figure 3 on the next page features the resulting list of play potentials of urban spaces. 


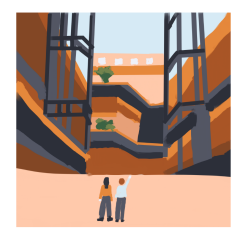

\#1 Admire the urban space

It can be fun to find unexpected, surprising, hilarious, visually stimulating things in the street. Designers should think about how their interventions could capture people's attention and provide an experience of wonder.

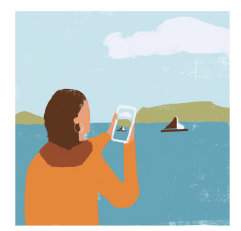

\section{Source: scraping social medi}

\#2 Stream exciting urban occurrences

People enjoy documenting interesting things they find around the city (e.g. beauty, action, surprises). Designers should think about how to allow people to document urban occurrences to share lived experiences and express themselves creatively.

\section{Source: scraping social media}

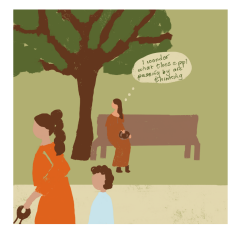

\section{\#4 Fill the gaps}

Fantasizing about things we know very little about can be fascinating and mind-absorbing. Designers should think about how their playful urban designs might help people tp imagine and fantasize with the backstories of others surrounding them.

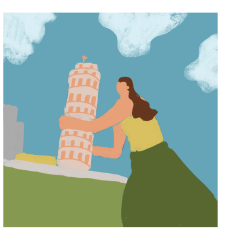

Source: play \& culture workshop

\section{\#6 Pose performatively}

People enjoy posing in strange ways around landmarks, statues, or out-of-place objects. Designers should think about how to enable and encourage playful kinds of posing experiences that are fun for both those who pose and those around them.

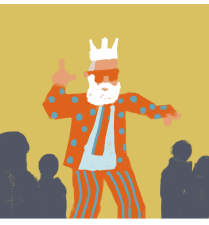

Source: scraping social media

\section{\#8 Customize the self}

Some people also enjoy wearing attention-grabbing clothing, wearables, lights, and costumes. Designer should think about how to facilitate experiences where people can enrich their public appearance.

\section{Source: scraping social media}

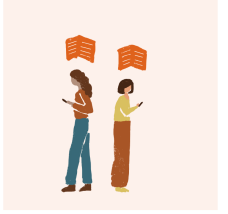

10 Connect with strangers

Urban spaces are social connection arenas. There is something playful about looking at other passers-by as treasures to discover. Designers should think about how to prompt people to accidentally meet strangers and initiate new connections.

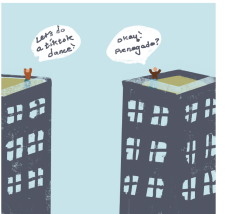

Source: scraping social media + play \& culture workshop

\section{\#12 Communicate at a distance}

It is fun to connect with others who are visible but at a distance, knowing that it'll be a brief and lightweight interaction. Designers should think about how to afford distanced and time-bounded social experiences that are exciting and fun.

Source: scraping social media

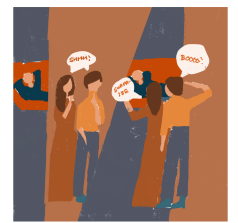

\section{\#14 Prank strangers}

If done safely, it can be fun to prank strangers. Designers should think about how to facilitate lightweight forms of urban pranking that are harmless (physically, emotionally, socially) and fun for both the pranker and the pranked.

\section{PLAY POTENTIALS OF URBAN SPACES}
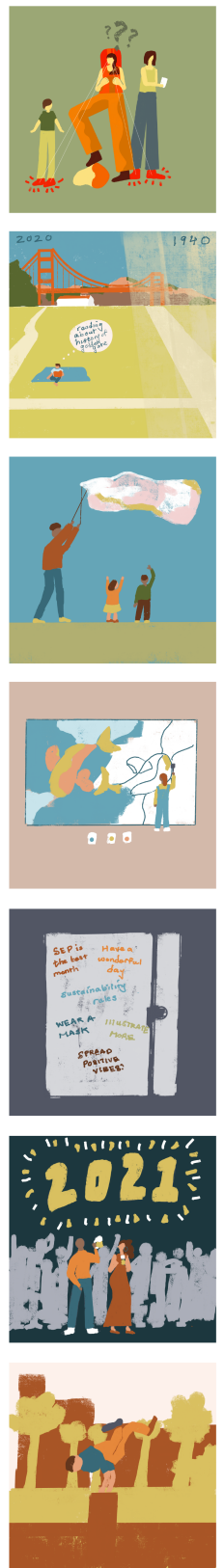

\section{\#3 Discover the invisible}

It can be fun to explore the hidden cultural subtext that conforms the socio-cultural fabric of a city. Designers should think about how to enable and encourage people to discover tacit urban knowledge to become familiar with an environment.

Source: play \& culture workshop

\section{\#5 Temporal decontextualization}

There is something exciting about learning about the real or imagined history of an urban space while physically present. Designers should think about how to enable people to share and learn the stories that took place in a city.

Source: play \& culture workshop

\section{\#7 Performative behaviors}

Some people enjoy doing things that are noticeable (e.g. silly, creative, or otherwise remarkable). Designers should think of ways to augment urban spaces in ways that people feel safe to be performative for their own enjoyment and to amuse others.

\section{Source: scraping social media}

\section{\#9 Hack the street}

Streets can be a canvas for personal expression: to make art, share ideas, and communicate through messages. Designers should think about how to enable citizens to appropriate the urban space and use it to advance their creative/expressive agendas.

Source: scraping social media + and play \& culture workshop

\section{\#11 Asynchronous communication}

There is an element of fun, mystery, and excitement in leaving messages on public spaces for other people to find. Designers should think about how to augment the urban space with technology that enables people to communicate asynchronously.

\section{Source: scraping social media}

\section{\#13 Massive celebrations}

There is something exhilarating in being part of a massive celebration. Designers should think about how to use technology to enable moments of collective celebration, bringing strangers momentarily together to cheer and share joy.

Source: scraping social media

\section{\#15 Urban challenges}

Some people enjoy self-imposed urban challenges, e.g. jumping on certain tiles, or crossing the street in $<\mathrm{n}$ seconds. Designers should think about how to afford small, spontaneous challenges that add a bit of spice to people's ordinary urban movements.

Figure 3: Our list of urban play potentials, identified through two interventions: scraping social media and a play $\&$ culture workshop. A plain text version of the list can be accessed at: https://bit.ly/fig3alt 


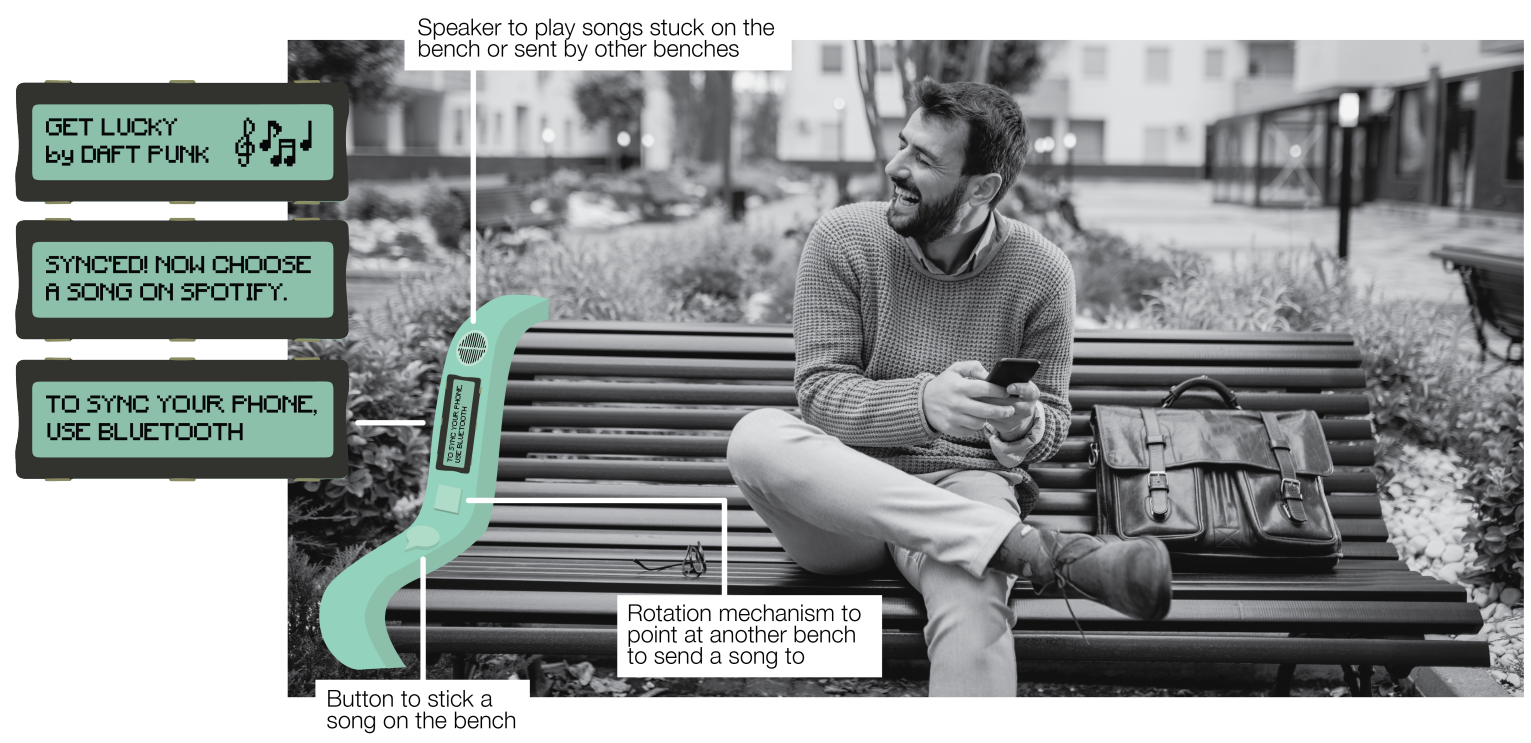

Figure 4: Mock-up of Share-a-song: a bench equipped with a "social sound system" that allows people to create and deliver ephemeral musical experiences to others around them.

\section{A CATALOG OF SPECULATIVE URBAN TECH}

To explore the inspirational value of our approach, we began to speculate about how future urban technology could respond to the play potentials we found in our contextual research. We began with a first round of ideation done independently by 6 designers over two weeks. We aimed to generate early ideas of technology-mediated urban experiences that embraced at least one play potential. We produced 25 ideas, which we collected on a slideshow ${ }^{3}$ and expanded at a subsequent brainstorming session.

Next, two designers examined the collection of early ideas to identify recurrent themes. We discussed their findings in another meeting, where we settled on a set of 7 emerging design directions that (1) we found interesting and (2) resonated with the findings from our contextual research. Then, we took two weeks to concretize each design direction into 1-2 urban technology design concepts, taking our collection of early ideas as a point of departure. We refined the resulting concepts at a final brainstorming meeting, discussing and extending each other's ideas. Throughout the process, we kept track of how the emerging ideas related to the play potentials from our contextual research ${ }^{4}$.

Once our design directions and illustrative technology concepts were ready, two designers mocked them up using graphic design tools. Inspired by [11], we created a Catalog of Speculative Playful Urban Technology Ideas ${ }^{5}$. We frame it as an annotated portfolio [28] of speculative design ideas highlighting interesting and socioemotionally desirable forms of technology-mediated urban play. Here we present the 7 design directions in our portfolio, using some of the associated technology ideas to illustrate them.

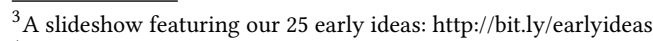

${ }^{4} \mathrm{~A}$ spreadsheet linking our ideas to the play potentials: http://bit.ly/ideaspotentials

${ }^{5}$ The full catalog: http://bit.ly/playfulurbantechcatalog
}

"Augmented infrastructure for authoring urban experiences” is our first design direction. It refers to urban infrastructure (e.g., a light pole, a bench, a façade. . .) that is augmented with digital technology (e.g., smart lighting, projectors, speakers...) and allows people to use a range of multimedia affordances to be creative and craft novel urban experiences-let it be for themselves or for others. Our catalog features two design ideas that illustrate this design direction. One of them is Share-a-song (Figure 4), an Internet of Things device that equips public benches with a "social sound system". The augmented benches allow people who sit on them to sync their phone, choose a song on Spotify, and either (a) send it to a nearby bench or (b) leave it on their bench to be enjoyed by the next person who sits there. One way or another, citizens can use their bench to craft short, ephemeral musical experiences for others around them, engaging in a rather unusual (i.e., asynchronous or physically distanced) interaction with people with whom they share the urban space (likely strangers).

"Parallel (in)visible realities", our second design direction, are augmented or mixed reality systems that enable citizens to experience fantastic realities that take place in parallel to the ordinary flow of urban life-and, as a result, to bond with others who also decide to experience them. Importantly, those parallel realities are only visible to those who use the system; that is meant to create a sense of community between users. An example of a technology that aligns with this design direction is A Mad Hatter's world (Figure 5), an "invisible" game that allows citizens to wear virtual hats that can only be seen through an AR app. People can create custom designs, choose from a set of pre-designed hats, or even steal someone else's hat idea and keep it for themselves. At the core of this design is the idea of helping people to bond and feel connected with others, even with strangers, in a lightweight and non-invasive manner: by being part of a somewhat silly community of mad hatters accessible only through a dedicated app. 


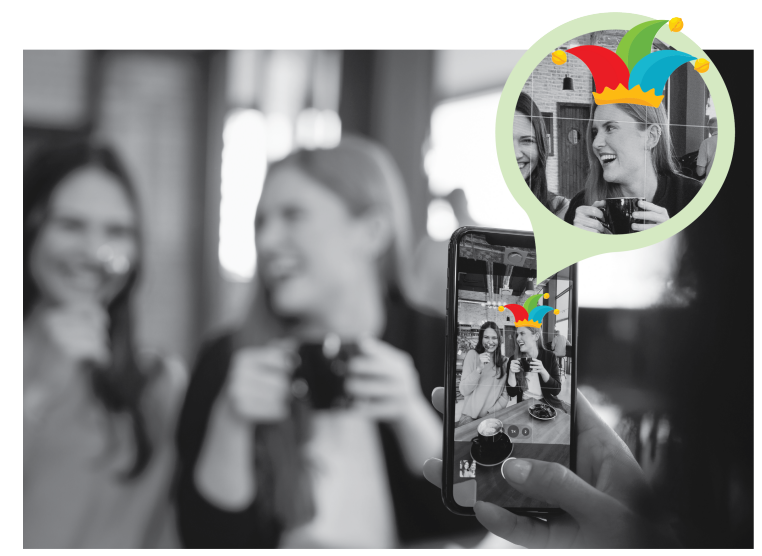

Figure 5: Mock-up of A Mad Hatter's world: a smartphone app that allows people to wear extravagant virtual hats and bond over that (somewhat silly) shared experience.

"Spontaneous instigators of strange(r) connections" are short, fast-paced invitations to social play placed in urban locations where people often wait or pass the time. They aim to create strange, silly, or otherwise unusual situations that invite citizens to do something unexpected during their wait in ways that encourage them to initiate new connections-even if ephemeral. Our catalog includes two design ideas under this theme. One of them is Dancing the light (Figure 6), a game augmenting pedestrians' experience of waiting at a traffic light. When the light is red, a song plays on a speaker, and a screen invites people to make dance moves matching the music. The more (and the better) people on both sides dance, the sooner the light will turn green. By providing people with that bait, this design hopes to encourage them to let go and submit to

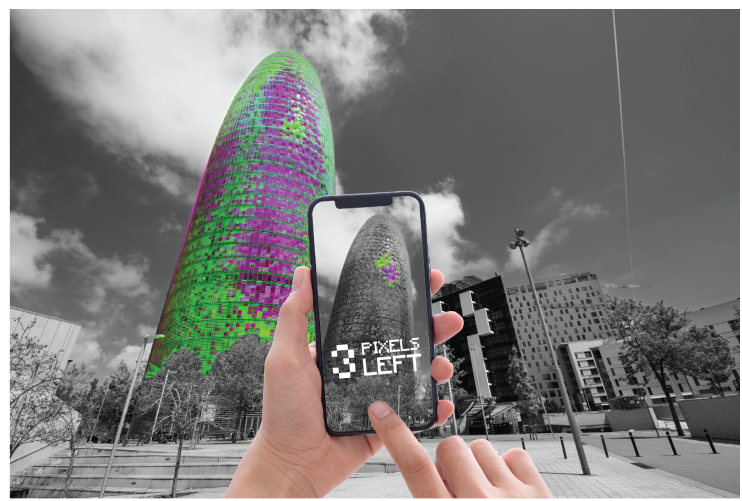

Figure 7: Mock-up of Building art, which turns a building's facade into a giant canvas for collective artistic expression.

a somewhat silly and unproductive ephemeral activity that will likely contribute to some shared laughter and fun. It is also meant to instill a sense of fellowship in strangers who happen to be at the same side of a crosswalk, and to help them to momentarily bond over the celebration of a win (or to playfully mourn a loss) in a race against people on the other side of the street. This idea is inspired by existing designs that playfully repurpose a city's traffic light poles (e.g., StreetPong [23]) and extends them by enabling people to interact in a group-using their bodies and the physical space rather than through a screen embedded in the light pole.

"Large scale urban toys", our fourth design direction, are interactive installations without an apparent purpose other than allowing people to experiment with them in lightweight, open-ended, momentary, and playful ways. Our catalog includes two design ideas related to this theme. One of them is Building art (Figure 7),

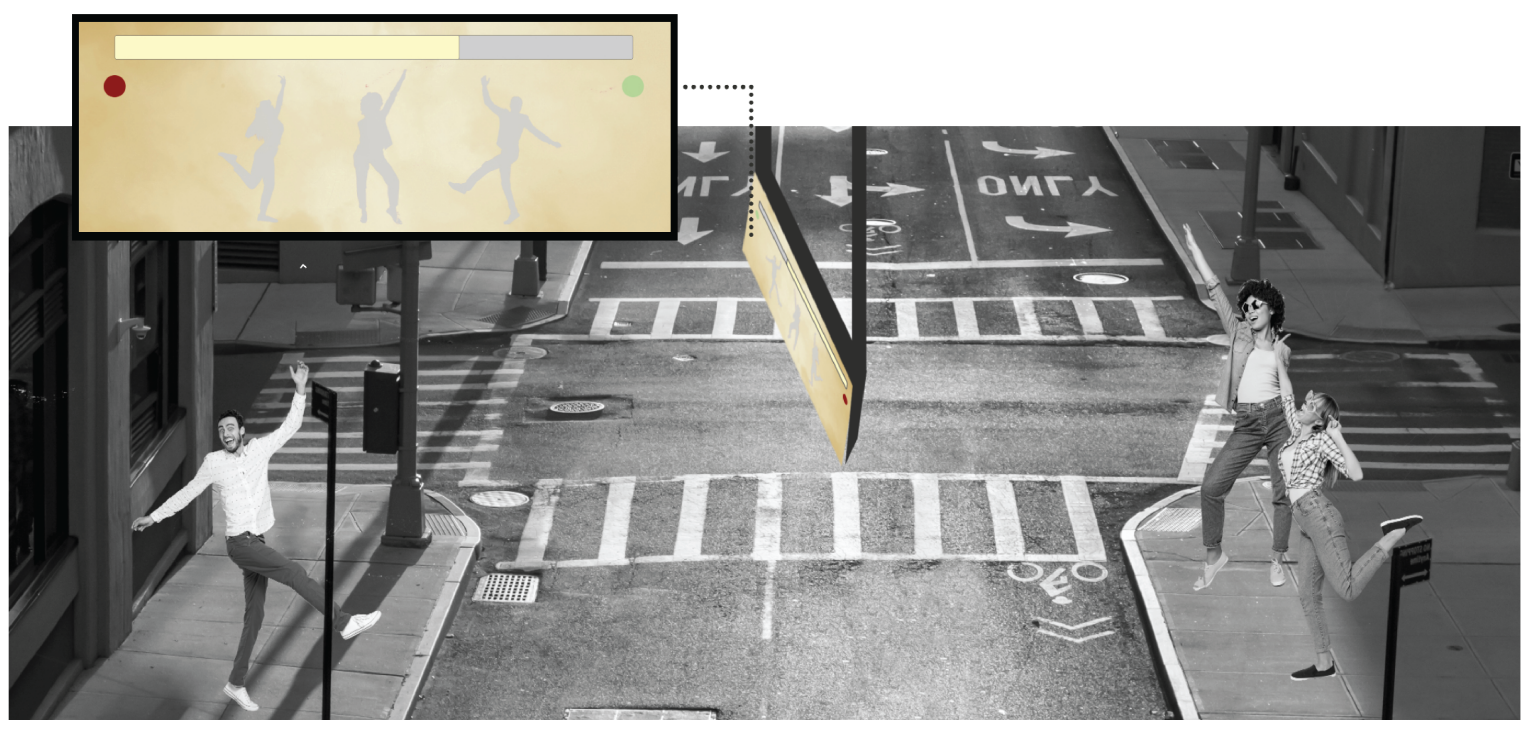

Figure 6: Mock-up of Dancing the light: an augmented traffic light that invites pedestrians on both sides of a crosswalk to let go and dance to a shared tune and rewards them by shortening the wait time based on their performance. 


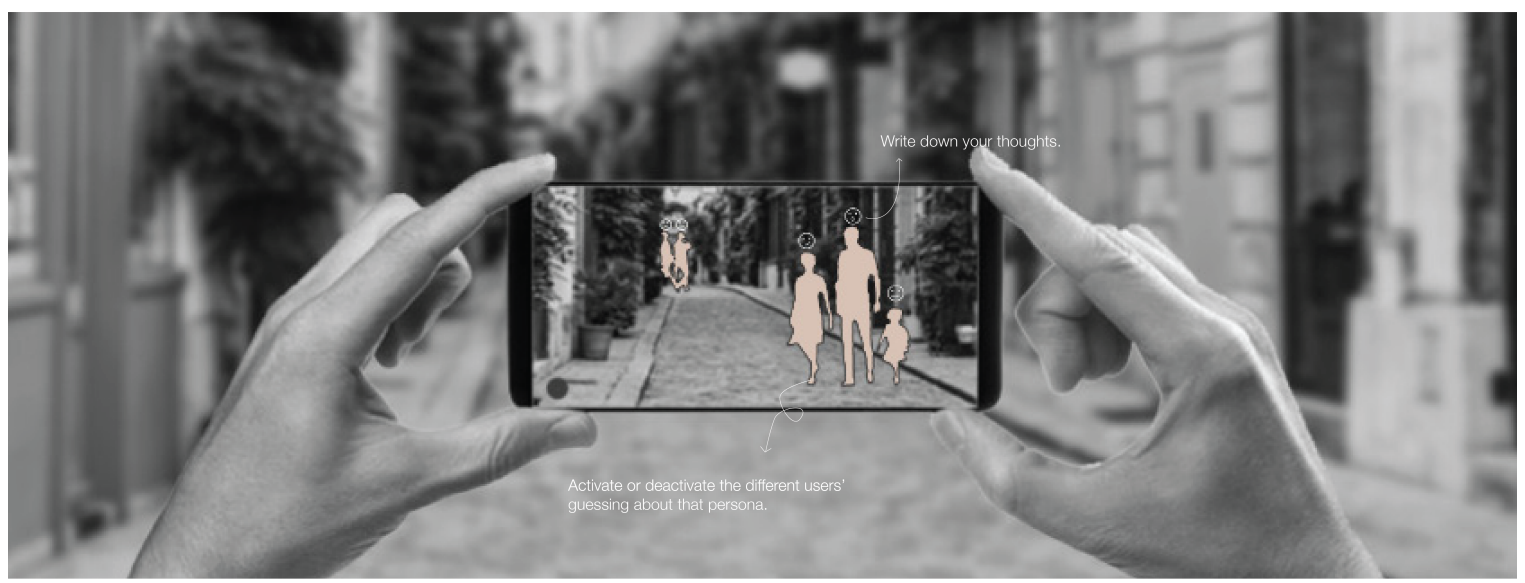

Figure 8: Mock-up of Silhouettes: an AR app and "unproductive" surveillance system that allows people to see the anonymous silhouettes of others who passed by where they currently are, and to build on that ambiguous and incomplete information to fantasize with the life stories of those silhouettes.

which equips a building's facade is with LEDs that citizens can appropriate artistically. The lights are connected to an app that allows people to change their color. Once a day, the facade opens up for some minutes so that people can create a new light composition. They can gather in front of it and paint 20 pixels each. The resulting ephemeral piece of collective art will be displayed for all citizens to enjoy until the canvas resets the next day.

"Portals of imagination" are technologies that afford experiences of fantasy, imagination, and wonder. They provide citizens with an ambiguous and deliberately incomplete story and invite them to fantasize and fill the gaps. Two ideas in our catalog exemplify this design direction. One of them is Silhouettes (Figure 8), an AR app and "unproductive" surveillance mechanism that allows citizens to see the silhouettes of people who were in that same space before, along with the system's guess of the silhouettes' emotional state. That way, citizens can fantasize about what happened earlier in the very space they are inhabiting and share their guesses as comments to gossip with other users of the app.

"Local lore modules" are systems that use digital technology to deliver local knowledge to visitors or newcomers-that is, information that is locally-produced and goes beyond the mainstream. For example, the technology may contain stories about the locals, about the fauna and the flora, or tips about the idiosyncrasies of the place. Locals can appropriate the system and populate it with the content of their creation. That content will be delivered to visitors to enable them to discover the place genuinely. Our catalog features two design ideas under this theme. One of them is the Scavenger Hunt Plaques (Figure 9), which can be placed anywhere within a city or neighborhood. Locals can populate the plaques with information relevant to their location: a thing they experienced in that place, a relevant story that is not commonly known... Visitors can scan the plaques and discover those things that are only known by locals, thereby learning stories that would not otherwise be accessible to them.

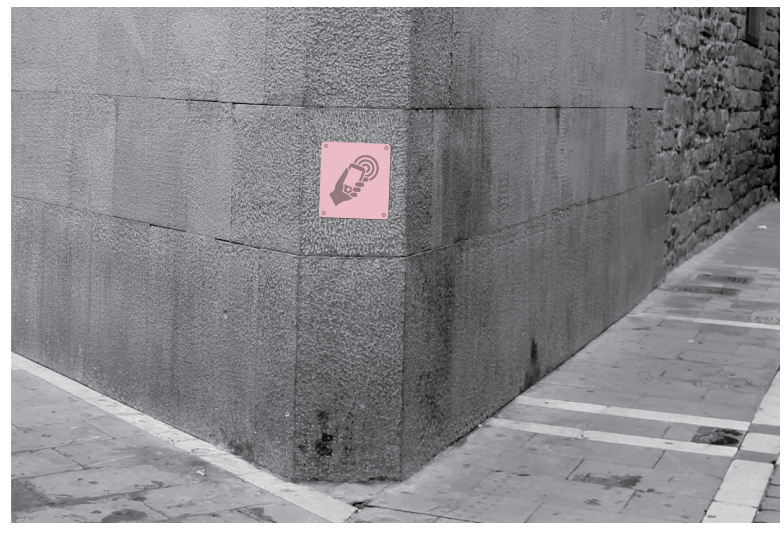

Figure 9: Mock-up of the Scavenger Hunt Plaques, which can be populated with local information by citizens so that visitors and newcomers can get to know a place beyond the surface.

“Shared canvases for collective grandeur”, our last design direction, are interactive installations that reflect the socio-emotional state of a city and allow citizens to be part of an emergent multimedia spectacle. Our catalog includes two ideas under this theme. One of them is the Fountain of whispers (Figure 10), a water fountain that reacts to the emotions of those surrounding it. Once a day, through a phone app, people can record a voice memo about their mood, about, or about something that happened to them, and then send it to the fountain. The fountain will use the data to dynamically react to the citizens' emotions, reflecting the city's mood by changing the water flow dynamics, by raising or lowering the temperature of the water, by modifying the color of the lights illuminating the water, or by using a range of ambient sounds. 


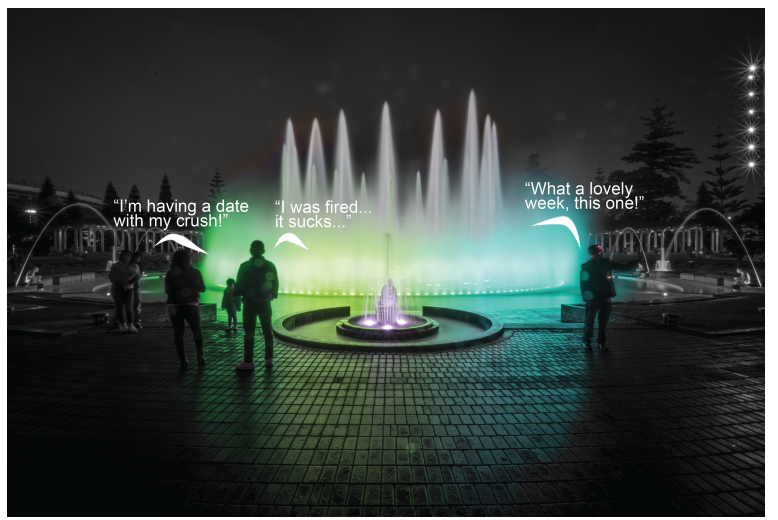

Figure 10: Mock-up of the Fountain of whispers, which creates a multi-sensory spectacle that reflects a city's emotional state.

\section{RELEVANCE, LIMITATIONS AND FUTURE WORK}

The design directions and concepts presented above contribute to an ongoing shift in values behind smart city innovation-arguably, a necessary one. We present our work as inspiration for designers interested in developing urban technology that contributes to shaping public spaces where individuals and communities can flourish-productively, yes, but also socially, emotionally, and culturally. Our ideas respond to urban experiences that, though they hardly are embraced in commercial smart city implementations, people seem to long for. Table 1 synthesizes how ideas in our catalog respond to the play potentials from our contextual research. However speculative, our early proposals can help designers to be mindful of playful and social practices people already engage with and enjoy within their city- in ways that they can support (rather than disrupt) the playful potential inherent in an urban space. Our provocations are: bottom-up (i.e., they respond to existing urban practices of average citizens) and socio-emotionally focused (i.e., they center on supporting rich, delightful urban experiences). As such, they challenge approaches to smart city innovation that, as [14][43] note, are often top-down, utilitarian, and techno-centric.

In particular, we see two ways our work can contribute to shaping socio-emotionally sensitive smart city futures. First, our catalog of speculative ideas and underlying design directions can inspire designers at the early stages of their work, focusing them on affording types of urban experiences that are socially, emotionally, and culturally desirable. Second, play potentials can help designers to examine their ideas (whether inspired by our catalog or not) and assess whether they respond to contextually grounded playful and social practices citizens long for. We hope combining these two will stimulate and make actionable smart city innovation that transcends utilitarianism and techno-determinism.

As early technology concepts, the ideas on our catalog are halfbaked and ambiguous. That could be seen as a limitation of our work: we have not yet prototyped or tested them in a naturalistic setting, and their actual impact remains unclear. While our work is by no means an advanced design proposal that can be readily implemented, we argue for its relevance as an inspirational starting point. It is an early provocation for designers to think differently about how technology-mediated urban experiences might be more playful, contextually meaningful, and socio-culturally sound.

We also acknowledge that previous research and artistic work has produced technologies that align with some of the ideas in our catalog, e.g., SelfieCafe [52], spread.gun [25], Urbanimals [39] or Hello Lamp Post [49], all described in Section 2. Other designs hold resemblances with some of our ideas. For example, one of our reviewers pointed out that the façade of the Hotel WZ Jardins in São Paulo [25] is somewhat similar to Building Art, as it changes color and can be interacted with via smartphone. In showing our catalog to other designers, we learned about other works similar

Table 1: Summary of ideas (and underlying design directions) included in our Catalog of Speculative Playful Urban Technology Ideas, linked to the play potentials they respond to. The ideas described in this paper are marked with an asterisk. An accessible version of the table, including the 13 catalog ideas and our early collection of 25 ideas, can be accessed at: http://bit.ly/table1alt

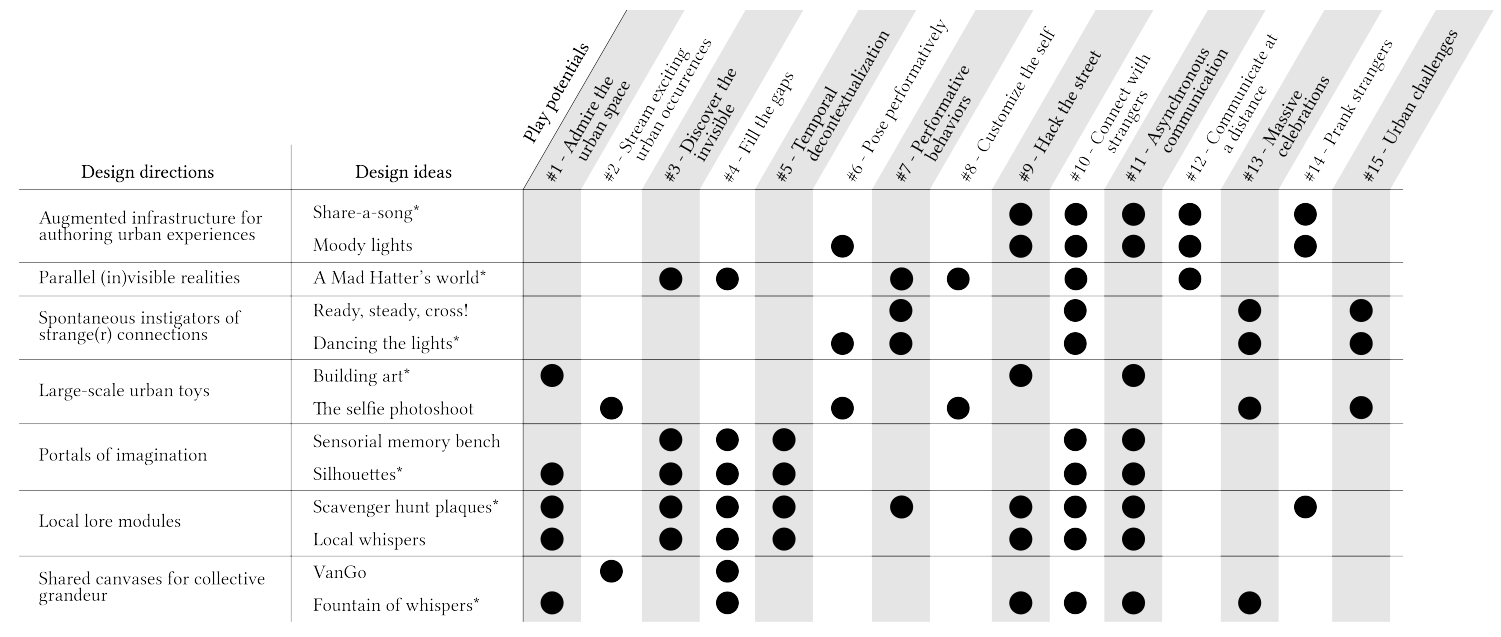


to ours (e.g., Shadowing [17], an interactive installation that, like Silhouettes, allows citizens to play with shadows from the past). We argue our work adds value despite those similarities: rather than claiming exclusivity over the idea of designing tech that playfully augments the city, we show our situated process of identifying playful practices people do in the public space and building on them to speculate how tech could respond to their playful potential. We suggest our approach can help designers to create technology that is grounded in people's playful cravings rather than on the designer's creative intuition-and that, as a result, is more likely to afford experiences that are contextually sound.

Further, while exceptionally playful urban technology works exist in research and artistic domains, the reality is that they still remain unusual when we look at commercial smart city implementations, where utilitarian approaches dominate [31]. We hope that our ideas, combined with other efforts such as those cited throughout this paper, contribute to challenging this trend. For example, some of our catalog ideas rethink the functionality of, and experience delivered by, urban navigation tools like Google Maps. Rather than trying to optimize people's urban movements, they support non-utilitarian agendas such as highlighting the richness of the city and its citizens (see Scavenger Hunt Plaques) or tracking people's movements to produce pieces of collaborative art (see VanGo, p. 27 of the catalog). Other ideas hint at how emerging technology could extend existing, pre-smart city urban infrastructure in ways that put people's wellbeing before urban efficiency. For example, Dancing the light augments traffic lights by (1) turning them into opportunities for social connection and (2) giving people a chance to (playfully) determine the length of their wait-aligning with recent moves towards privileging pedestrians over cars (e.g., [48]). Our ideas also foreground ways in which dedicated urban play could be more smoothly integrated into the ordinary flow of urban life in ways that enrich the socio-cultural fabric of a city. For example, Silhouettes (p. 8) extends location-based games such as Pokémon Go by centering the player's attention towards their environment and the other citizens with whom they share it, instead of inviting them to a fantastic virtual reality that has little to do with the ordinary flow of the city life.

In addition to its inspirational potential, we also see our catalog as a valuable prompt to engage diverse stakeholders to co-imagine the playful future of their city. Our ideas illustrate a breadth of possible design directions within a design space most average citizens are unfamiliar with. By making those design directions tangible and relatable, we can help non-experts to be involved in shaping the foundations of smart city innovation in ways that both their pragmatic needs, their values, and their playful cravings are considered. That is an important consideration, as the ideal of a playable city brings about implicit tensions: while making the urban space more playful and enjoyable for some, it may create accessibility constraints for others or invade the privacy of citizens who would rather not be a part of the digital sphere. We are currently in the process of experimenting with the catalog as a tool to facilitate multi-stakeholder conversations about those issues, engaging average citizens, experts, and policy-makers alike to respond to, extend, and rethink our catalog. We see the catalog not as a set of finalized design proposals but rather as a prompt to provoke these kinds of diverse and imaginative discussions of what the playful future of our cities should look like. In the future, we will disseminate the outcomes of our multi-stakeholder engagements based on the catalog. We will also build prototypes of the technology concepts that better resonate with our participants' ideas, further developed through the lens of people's diverse understandings of what a playable city should be.

Another limitation of our contribution is that our findings might not necessarily be universally applicable. The play potentials we identified in our contextual research are grounded in existing practices we observed in specific urban settings. Some might not apply beyond the contexts where they were found, and there could be many more play potentials on our list had we explored other urban settings. To use our list of play potentials, design directions, and speculative ideas, designers should explore if and how they apply to the context targeted by their project. As noted by [9], there is a lot of value in designing for the particular, as it "can enable us to capture the richer and more complex nuances of a particular situation or user, hence also directly challenging the assumptions we make as researchers". Taking our work as a point of departure, designers can use SPD [1] and other participatory methods to further understand what kinds of urban experiences their target citizens long for. That will enable the design of urban technologies that realize the playful potential of the targeted context. This paper provides some guidance for how to do that: it shows how we leveraged two SPD methods to chase play potentials of urban spaces and use them to drive design (see Section 3). We hope that these strategies empower designers to identify design opportunities for urban play in their targeted design context, adapt our proposed play potentials and design directions, and find new ones that better respond to the specific idiosyncrasies of the cities they design for.

\section{CONCLUSION}

In this paper, we presented an annotated portfolio of speculative playful urban technology concepts that build on and illustrate in designerly ways the findings from our research into the playful potential of urban spaces. Our speculative ideas highlight a number of urban play forms that people seem to long for and propose ways of responding to them by design. Though clearly speculative, we argue that our work can inspire the design of future smart city technology that pays more attention to the social, emotional, and cultural potential of our cities. We see value in sharing the work now, even if it is still part of an ongoing design research process; it will (1) spark multistakeholder conversations about what playable cities should be in the future, (2) contribute to an ongoing shift in values behind smart city innovation, and (3) provide inspiration, both conceptual and methodological, to support that shift. We hope that our contribution provokes designers to embrace increasingly playful approaches when designing urban technology and provides inspirational starting points that make that move more actionable.

\section{REFERENCES}

[1] Ferran Altarriba Bertran, Jared Duval, Katherine Isbister, Danielle Wilde, Elena Márquez Segura, Oscar Garcia Pañella, and Laia Badal León. 2019. Chasing Play Potentials in Food Culture to Inspire Technology Design. In Extended Abstracts of the Annual Symposium on Computer-Human Interaction in Play Companion Extended Abstracts (CHI PLAY '19 Extended Abstracts). Association for Computing Machinery, New York, NY, USA, 829-834. DOI:https://doi.org/10.1145/3341215. 3349586 
[2] Ferran Altarriba Bertran, Elena Márquez Segura, Jared Duval, and Katherine Isbister. 2019. Chasing Play Potentials: Towards an Increasingly Situated and Emergent Approach to Everyday Play Design. In Proceedings of the 2019 on Designing Interactive Systems Conference (DIS '19). Association for Computing Machinery, New York, NY, USA, 1265-1277. DOI:https://doi.org/10.1145/3322276. 3322325

[3] Ferran Altarriba Bertran, Elena Márquez Segura, and Katherine Isbister. 2020 Technology for Situated and Emergent Play: A Bridging Concept and Design Agenda. In Proceedings of the 2020 CHI Conference on Human Factors in Computing Systems (CHI '20). Association for Computing Machinery, New York, NY, USA, 1-14. DOI:https://doi.org/10.1145/3313831.3376859

[4] Ferran Altarriba Bertran, Laia Turmo Vidal, Ella Dagan, Jared Duval, Elena Márquez Segura, and Katherine Isbister. 2020. Chasing Play with Instagram How Can We Capture Mundane Play Potentials to Inspire Interaction Design? In Extended Abstracts of the 2020 CHI Conference on Human Factors in Computing Systems (CHI EA '20). Association for Computing Machinery, New York, NY, USA, 1-8. DOI:https://doi.org/10.1145/3334480.3382913

[5] Stefano Andreani, Matteo Giacomo Maria Kalchschmidt, Roberto Pinto, \& Allen Sayegh. 2019. Reframing technologically enhanced urban scenarios: A design research model towards human centered smart cities. Technological Forecasting and Social Change, 142, 15-25.

[6] Charbel Aoun. 2013. The smart city cornerstone: Urban efficiency. Published by Schneider electric

[7] Francesco Paolo Appio, Marcos Lima, \& Sotirios Paroutis. 2019. Understanding Smart Cities: Innovation ecosystems, technological advancements, and societal challenges. Technological Forecasting and Social Change, 142, 1-14.

[8] Jon Back, Elena Márquez Segura, \& Annika Waern. 2017. Designing for Trans formative Play. ACM Trans. Comput.-Hum. Interact. 24, 3: 18:1-18:28. https //doi.org/10.1145/3057921

[9] Olav W. Bertelsen, Susanne Bødker, Eva Eriksson, Eve Hoggan, and Jo Vermeulen 2018. Beyond generalization: research for the very particular. interactions 26,1 (January - February 2019), 34-38. DOI:https://doi.org/10.1145/3289425

[10] Scott L. Brown. 2009. Play: How it shapes the brain, opens the imagination, and invigorates the soul. Penguin.

[11] Barry Brown, Julian Bleecker, Marco D'Adamo, Pedro Ferreira, Joakim Formo Mareike Glöss, Maria Holm, Kristina Höök, Eva-Carin Banka Johnson, Emil Kaburuan, Anna Karlsson, Elsa Vaara, Jarmo Laaksolahti, Airi Lampinen, Lucian Leahu, Vincent Lewandowski, Donald McMillan, Anders Mellbratt, Johanna Mercurio, Cristian Norlin, Nicolas Nova, Stefania Pizza, Asreen Rostami, Mårten Sundquist, Konrad Tollmar, Vasiliki Tsaknaki, Jinyi Wang, Charles Windlin, and Mikael Ydholm. 2016. The IKEA Catalogue: Design Fiction in Academic and Industrial Collaborations. In Proceedings of the 19th International Conference on Supporting Group Work (GROUP '16). Association for Computing Machinery, New York, NY, USA, 335-344. DOI:https://doi.org/10.1145/2957276.2957298

[12] Barry Brown and Oskar Juhlin. 2015. Enjoying machines. Mit Press.

[13] Roger Caillois. 2001. Man, play, and games. University of Illinois Press.

[14] Ignasi Capdevila \& Matias Zarlenga. 2015. Smart city or smart citizens? The Barcelona case. The Barcelona Case (March 26, 2015).

[15] Center for the Living City (CLC). (N.d.) Jane Jacobs. Accessed on May 7, 2020 at https://centerforthelivingcity.org/janejacobs\#jane-and-the-center

[16] Yoram Chisik, Ferran Altarriba Bertran, Marie-Monique Schaper, Elena Márquez Segura, Laia Turmo Vidal, and Danielle Wilde. 2020. Chasing play potentials in food culture: embracing children's perspectives. In Proceedings of the 2020 ACM Interaction Design and Children Conference: Extended Abstracts (IDC '20). Association for Computing Machinery, New York, NY, USA, 46-53. DOI:https: //doi.org/10.1145/3397617.3398062

[17] Chomko and Rosier. 2014. Shadowing. Accessed on March 29, 2021 at https: //www.playablecity.com/projects/shadowing/

[18] Mihály Csikszentmihalyi. 1997. Finding flow: The psychology of engagement with everyday life. Basic Books.

[19] Renata Paola Dameri \& Camille Rosenthal-Sabroux. (Eds.). 2014. Smart city: How to create public and economic value with high technology in urban space. Springer.

[20] Bernie DeKoven. 2013. The Well-Played Game: A Player's Philosophy. The MIT Press, Cambridge, MA, USA

[21] Michiel de Lange. 2019. The playful city: Citizens making the smart city. The Playful Citizen, 349.

[22] Katerina Diamantaki, Charalampos Rizopoulos, Vasileios Tsetsos, Iulianos Theona, Dimitris Charitos, \& Nikos Kaimakamis. 2013, July. Integrating game elements for increasing engagement and enhancing User Experience in a smart city context. In Intelligent Environments (Workshops) (pp. 160-171).

[23] Sandro Engel and Amelie Künzler. 2012. StreetPong. Retrieved on March 28, 2021 from http://www.streetpong.info

[24] Engineering and Physical Sciences Research Council (EPSRC). (N.d). The Equator Project. Accessed on May 7, 2020 at https://web.archive.org/web/20120702141940/ http://www.equator.ac.uk/

[25] Estudio Guto Requena. 2015. Light creature: interactive facade as a visual sound scape reflection of São Paulo’s iconic avenue. Accessed on March 29, 2021 at https://en.gutorequena.com/lightcreature-en/

[26] Patrick Tobias Fischer, Christian Zol̈lner, Eva Hornecker. 2010. VR/Urban: Spread.gun-design process and challenges in developing a shared encounter for media facades. In: Proceedings of British HCI 2010, BCS eWiC series, pp. 289-298. British Computer Society/ACM NY

[27] Oscar García, Pablo Chamoso, Javier Prieto, Sara Rodríguez, \& Fernando de la Prieta. 2017, June. A serious game to reduce consumption in smart buildings. In International Conference on Practical Applications of Agents and Multi-Agent Systems (pp. 481-493). Springer, Cham.

[28] Bill Gaver and John Bowers. 2012. Annotated portfolios. interactions 19, 4 (July + August 2012), 40-49. DOI:https://doi.org/10.1145/2212877.2212889

[29] Anni Gentes, Aude Guyot-Mbodji, \& Isabelle Demeure. 2010. Gaming on the move: urban experience as a new paradigm for mobile pervasive game design. Multimedia systems, 16(1), 43-55.

[30] René Glas, Sybille Lammes, Michiel de Lange, Joost Raessens, and Imar de Vries (eds.). 2019. The Playful Citizen. Amsterdam University Press.

[31] Giuseppe Grossi \& Daniela Pianezzi. 2017. Smart cities: Utopia or neoliberal ideology?.Cities, 69, 79-85

[32] Greg Guest, Kathleen M. MacQueen, \& Emily E. Namey. 2011. Applied thematic analysis. sage publications.

[33] Deepi Harish. 2017. \#FoodPorn Is Changing The Way Millennials Eat. In Huffington Post. Published on March 24th, 2017. Accessed on October 23rd, 2018 at https:// www.huffingtonpost.ca/deepi-harish/foodporneating-habits_b_15574714.html

[34] Johan Huizinga. 1950. Homo Ludens: A Study of the Play Element in Culture. Beacon Press.

[35] Katherine Isbister. 2016. How games move us: Emotion by design. MIT Press.

[36] Katherine Isbister, Elena Márquez Segura, \& Edward F. Melcer. 2018. Social Affordances at Play: Game Design Toward Socio-Technical Innovation. In Proceedings of the $2018 \mathrm{CHI}$ Conference on Human Factors in Computing Systems (CHI '18). ACM, New York, NY, USA, Paper 372, 10 pages. DOI: https: //doi.org/10.1145/3173574.3173946

[37] Dhruv Khullar. 2016. How social isolation is killing us. New York Times, Dec 22, 2016.

[38] Suzanne Kirkpatrick, Nien Lam, \& Jamie Lin. 2011. Commons: A game for urban communities to improve their city through citizen stewardship. Accessed on September 24, 2020 at http://civictripod.com/civictripod.com/games/commons/ index.html

[39] Laboratory for Architectural Experiments. 2015. Urbanimals. Accessed on March 29, 2021 at http://lax.com.pl/portfolio_page/urbanimals/

[40] Dale Leorke \& Marcus Owens. (Eds.). 2020. Games and Play in the Creative, Smart and Ecological City. Routledge.

[41] Andrés Lucero, Evangelos Karapanos, Juha Arrasvuori, and Hannu Korhonen. 2014. Playful or Gameful? creating delightful user experiences. interactions 21, 3 (May-June 2014), 34-39. DOI: https://doi.org/10.1145/2590973

[42] Maria-Lluïsa Marsal-Llacuna, Joan Colomer-Llinàs, \& Joaquim Meléndez-Frigola. 2015. Lessons in urban monitoring taken from sustainable and livable cities to better address the Smart Cities initiative. Technological Forecasting and Social Change, 90, 611-622.

[43] Luca Mora, Roberto Bolici, \& Mark Deakin. 2017. The first two decades of smartcity research: A bibliometric analysis. Fournal of Urban Technology, 24(1), 3-27.

[44] Evgeny Morozov. 2013. To save everything, click here: The folly of technological solutionism. Public Affairs.

[45] Evgeny Morozov \& Francesca Bria. 2018. Rethinking the smart city. New York: Rosa Luxemburg Stiftung.

[46] Niantic. (N.d). Pokémon Go. Accessed on May 7, 2020 at www.pokemongo.com

[47] Anton Nijholt (ed.). 2017. Playable cities. Singapore: Springer

[48] Feargus O'Sullivan. 2020. Barcelona Will Supersize its Car-Free 'Superblocks'. In bloomberg.com. Accessed on January 18, 2021 at https://www.bloomberg.com/ news/articles/2020-11-11/barcelona-s-new-car-free-superblock-will-be-big

[49] PAN Studio, T. Armitage, and G. Galik. 2013. About Hello Lamp Post. Accessed on March 29, 2021 at http://www.hellolamppost.co.uk/about

[50] Michel Peeters, Carl Megens, Elise van den Hoven, Caroline Hummels, and Aarnout Brombacher. 2013. Social stairs: taking the piano staircase towards longterm behavioral change. In International Conference on Persuasive Technology (pp. 174-179). Springer, Berlin, Heidelberg.

[51] Manaswi Saha, Kotaro Hara, Soheil Behnezhad, Anthony Li, Michael Saugstad, Hanuma Maddali, Sage Chen, and Jon E. Froehlich. 2017. A Pilot Deployment of an Online Tool for Large-Scale Virtual Auditing of Urban Accessibility. In Proceedings of the 19th International ACM SIGACCESS Conference on Computers and Accessibility (ASSETS '17). Association for Computing Machinery, New York, NY, USA, 305-306. DOI:https://doi.org/10.1145/3132525.3134775

[52] Tony Salvador, John W. Sherry, \& Alvaro E. Urrutia. 2005. Less cyber, more café enhancing existing small businesses across the digital divide with ICTs. Inf. Technol. Dev. 11(1), 77-95 (2005).

[53] Ben Schouten, Gabriele Ferri, Michiel de Lange, and Karel Millenaar (eds.). 2017. Games as strong concepts for city-making. In Playable Cities (pp. 23-45). Springer, Singapore. 
[54] John Sharp and David Thomas. 2019. Fun, Taste, \& Games: An Aesthetics of the Idle, Unproductive, and Otherwise Playful. MIT Press.

[55] Miguel Sicart. 2014. Play matters. MIT Press.

[56] Bhagya Nathali Silva, Murad Khan, \& Kijun Han. 2018. Towards sustainable smart cities: A review of trends, architectures, components, and open challenges in smart cities. Sustainable Cities and Society, 38, 697-713.

[57] Henrik Sproedt. 2012. Play. Learn. Innovate. Books on Demand.

[58] Staff, J. 2014. Why is everyone so busy. The Economist. Retrieved from http://www. economist.com/news/christmasspecials/21636612-time-povertyproblempartly-perception-and-partly-distributionwhy

[59] Jaakko Stenros. 2014. In defence of a magic circle: the social, mental and cultural boundaries of play. Transactions of the Digital Games Research Association, 1(2).
[60] Jaakko Stenros. 2015. Behind games: Playful mindsets and transformative practices. The Gameful World: Approaches, Issues, Applications, MIT Press, Cambridge.

[61] Quentin Stevens. 2007. The ludic city: exploring the potential of public spaces. Routledge.

[62] Pekka Sulkunen. 2009. The saturated society: Governing risk \& lifestyles in consumer culture. Sage Publications.

63] Brian Sutton-Smith. 2009. The ambiguity of play. Harvard University Press.

[64] Mattia Thibault. 2019, January. Towards a typology of urban gamification. In Proceedings of the 52nd Hawaii International Conference on System Sciences.

[65] Stephen J. Vodanovich. 2003. On the possible benefits of boredom: a neglected area in personality research. Psychology and Education: An Interdisciplinary fournal.

[66] Eric Zimmerman. 2015. Manifesto for a ludic century. The gameful world: Approaches, issues, applications. MIT Press. 